\title{
Evaluating protocols of energy effectiveness and coverage in Wireless Sensor networks
}

\author{
Mrs. Shabda Dongaonkar \\ Phd Scholer, Koneru Lakshmaiah Education Foundation, \\ CSE, Vijaywada, Andhra Pradesh, 522302, India, \\ Shabda13@gmail.com \\ https://www.kluniversity.in \\ Dr.N.Srinivasu \\ Professor, Koneru Lakshmaiah Education Foundation, \\ CSE, Vijaywada, Andhra Pradesh, 522302, India \\ srinivasu28@kluniversity.in \\ https://www.kluniversity.in
}

\begin{abstract}
In each and every filed of applications, Wireless sensor networks are prominently used almost in all areas like military surveillance, home based automation, agriculture based, health care industries sensor network plays a vibrant role. In wireless sensor networks, performance of application depends on sensor's working, lifetime of sensors communication among sensors. There are two crucial issues in WSN as energy efficiency and coverage of area in which sensors are working. In proposed implementation, comparison of two protocols which are used for coverage and energy parameters. Simulation results shows that proposed protocol which is Preservation Efficiency and interactive Connectivity (PEAIC) gives more efficiency and performance as compared to CCOSS protocol. There are nine parameters of comparison in which graphical results shows performance of each protocol. The proposed protocol gives more efficiency and coverage which is very useful for wireless sensor networks applications.
\end{abstract}

Keywords: Wireless sensor networks, energy efficiency, coverage, Sensor node.

\section{Introduction}

In wide range of applications, role of wireless sensor networks is very significant. Either on the health care industries or in industrial applications sensors are there. It is very much essential that whenever we are talking about wireless sensor networks, it is must to know that how sensors are sensing something, communicating with each other and for this sensing and communication these sensors need some sort of energy which will be given by batteries but as the capacities of battery is not more because it should follow the characteristics of tiny in nature which is one of the requirement of wireless sensor networks design.

For utilizing available resources in wireless sensor networks proficiently, so many hierarchical clustering methods are used. The aim is to get more lifetime and increased efficiency of power. In this method, clustering is used for sensor nodes.

Removal of repetitive messages for the development of proficient clusters and choice of cluster head is also vital.[1]

In the paper, review is taken for the group-based and grid-based methods creation, energy spent, and network lifespan. Besides, this the design concerns and research challenges for doing research of hierarchical approaches have been discussed.

Based on the models of wireless sensor networks, design and construction issues were discussed for type of collection of data, and aggregate that data again. What is main reason to use clustering and routing in the enhancement of life span of network. By considering types as structure and structure-free designs the usefulness and demerits of the routing and clustering techniques are discussed here. [2]

The analysis was taken regarding coverage related protocols in networks where sensors are working. For the sake of reducing power consumed in the network, some of the sensor nodes with intersecting sensing area are switched off. [3]

Investigation of key coverage optimization protocols taken for this reason.Wireless sensor network is a profoundly powerful organization condition and a class of remote organization extraordinarily intended for checking. Enormous number of uses, for example, military frameworks, object following, checking, catastrophe revealing are planned on head of the WSN. These are constant applications and produce touchy information or pressing information. This touchy data needs to impart dependably. Exact conveyance of delicate data has straight influence on the general exhibition of the framework. Accomplishing unwavering quality and clog free correspondence is significant for the WSN.[15] 
Quality protection is perhaps the greatest test to the fruitful WSNs since the little extremely restricted asset hubs, for example, vitality, memory space| just as correspondence and calculation abilities.Endless checkups have be done in diverting vitality proficient calculations or conventions for WSNs.. At first of all First, prompt conveyance is utilized under little scope while multi-bounce network communication network is utilized under mass. It is need to discover the Which components impact the communication way. Multi-jump concur sending more vitality effective than usually communicating When the normal lone source to objective separation is huge. However, what to look like for the ideal jump number all together that the overall vitality utilization is ostensible isn't all around handled. [17]

Basic issues which brings the field into spotlight among scientists. The WSN comprise of set of sensor hubs, used to gather enormous total of information in powerful evolving condition. The size of the sensor cores is little, low battery powered, minimum cost, and arbitrarily hub organization. There are various of issues which can without much of a stretch breakdown the network, one of the fundamental issues to be noted in the environment is Coverage. It is a main point of contention in WSN ,mainly utilized for assessing the Quality of Service (QoS)of an organization.[12]

The activity of the hubs in remote impromptu organizations rely upon the battery control and have restricted vitality assets. The damage of some transitional hubs may cause significant topological changes, debilitate the organization activity, and affect the lifespan of the organization. This makes vitality proficiency a key worry in guaranteeing framework versatility. Vitality effective directing issues are significant in MANET dynamic condition. [14]

Vitality must to be ideally used with the goal that the hubs will play out their activity adequately. Fault resistance is a noteworthy property of impromptu organization, which guarantees the constancy of the assets. In this paper, a novel issue open minded multi way steering convention is proposed to lessen the bundle misfortune because of course breakage, which utilizes another course find and support system. It utilizes elective course to re send the information at whatever point a middle of the road hub doesn't ready to advance it, because of connection disappointment or hub disappointment.

The consideration in WSNs should be to such an extent that the essentialness of the sensors would be the base to rise the lifespan of the framework. To assess the region consideration issue in remote sensor organizes, the Particle Swarm Optimization and Differential Evolution computations which have separated the district incorporation issue in networks with sensors. To arrange the practicability of the hybrid model in a comparative condition a PSO figuring is executed. As per outcomes of the assessments it shows that the cream computation has made additional advancement in the age of the framework and extra improved usage of the essentialness of the sensors by updating the consideration of the sensors interestingly with PSO.[4]

The incorporation profitability is improved by reducing the over-lapping consideration degree achieved from the 3-Sym system, which is named as the decent region progression method (O-Sym). This considers consideration abundance inside the particular zone, expressly sensor's area the all out related incorporation is practiced by the generation results show that we accomplish not right over the all out checked region it should also observe the sensors which are not covered yet and interested.[5]

The consideration capability is evaluated on a proportion of the genuine incorporation and the supported spread age similar to degree and space. The three-adjusted domain technique (3-Sym) picks and starts certain sensors inside even regions of each. the amount of dynamic sensors is reduced and moreover the covering consideration can be diminished to gravitate toward to the perfect incorporation degree.[5]

The inspiration behind arbitrary arrangement remote sensor organization (WSN) is introduced in the paper. At that point analysed the all-inclusive brush needle model and bunch based ECNM for irregular arrangement of WSN is clarified with representations. The numerical investigation of ECNM and group based ECNM are clarified as far as correspondence and capacity overhead is done. The reproduction condition is given and closed the presentation assessment of ECNM and CECNM as far as boundaries, for example, correspondence cost, vitality utilization, throughput, delay, parcels conveyed, loss of packets.[20]

The presented paper will give a likelihood based K-inclusion control approach (PKCCA) in seeing of threedimensional space with equivocalness between the characteristic implausibility and vigorous flaw acknowledgment ability and the worth of remote sensor systems. The whole locale of perception is to be protected with the assistance of $\mathrm{K}$ sensors having the likelihood as $\mathrm{T}$. For deciding the best position of sensors.

A lattice dissemination is acquainted alongside covetous heuristic. The technique proposed in the paper will be finished when a current maximum breaking point on the quantity of sensors is broadened, or the assignment for inclusion is finished. The system we actualize partners it against conventional uniform arrangement alongside arbitrary sending. The outcomes got give a reasonable sign that PKCCA utilizes a decreased measure of sensors to finish the indistinguishable inclusion task or the alike sensors to impact predominant inclusion degree.[6] 


\section{Related work}

For getting idea of most widely used protocols for energy efficiency, we have to see Information securing from the environmental factors without human inclusion is a basic examination point. This issue can be settled by WSN (Wireless Sensor Network) which is more worthwhile and takes out the wired framework. For this paper, the WSN is sent with cutting edge sensors, which are minimized in size and are more affordable. For viable information transmission among hubs, a few hubs are chosen as center points, which are little in estimate and vary from one another. These centers are chosen dependent on how successfully the vitality is devoured. The coordinating shows, accordingly, must ensure less vitality use. Despite the point is that gathering of information using fixed center points is basic, collecting information from compact center points is problematic. This issue is settled by the convention shows like LEACH and PEGASIS that work uncommonly for fixed centers, however issue happens in choosing convention which is best reasonable for a specific application. This paper presents an itemized survey over the ideas of LEACH and PEGASIS and figures out which convention is more effective in social event data and guarantees less loss of data.[18]

For head determination with awareness of energy, GAL-LF introduced in networks with sensors. In consideration for the points of lifetime, departure and deferral of hubs of the sensors, recreation of the planned work was done. By seeing uniform energy and active centers of the selected cluster head again rebuilding took place. By showing differentiation of some of the conventional models like GA,PSO,ABC,GSO,ALO and CSbased it has been proved. [19]

It has been observed from Neighbor based cluster Location Aware Routing that it gives better conveyance rate with higher accuracy. It consists of mainly three states in the first state ,more sign quality is there as compare with bunch area. While in the second state, with more expansion fields, neighbor hub table is prepared. These fields are reliability, probability of productive transmission of packages and deferral. The back off clock is evaluated to revive the table inside a periodical time. In last stage, territory status manage is resolved to fabricate region precision and to enlarge the pack movement extent. In perception of the diversion results, the proposed plan NCLAR achieves high region precision, more bundle conveyance extent, less overhead, less delay and high association lifetime.[21]

The investigation of maximum remote sensor frameworks is stressed over imperativeness viability; compose life time and limitation issues of sensor middle points.

Sensor center points need to contact with the going with resource impediments:

1) Power Consumption: Wireless frameworks have a limited reserve of working; imperativeness guarding is a huge measure for system structure.

2) Communication: As remote frameworks have confined transmission limit, it induces to obliged trustworthiness, low nature of organization like high idleness, high contrast ,high packaging hardship. Besides, some security exposures like renouncing of administration, sticking, etc.

3) Computation: Because of compelled power and memory use , restrictions are there on data planning counts.

4) Uncertainty in estimated parameters: Node glitch may cause hasty range of information and sending this wrong information.

Considering above purposes of remote sensor arrange, numerous conventions were anticipated for vitality protection, restriction issues in WSN.

After studying all the above protocol studies of wireless sensor networks, the need of comparison regarding some of the very much vital parameters is there, so we will consider the below parameters which will give results useful for getting more lifetime and coverage in WSN.

2.1 Steps of the algorithm in PEAIC:

1. Start cluster deployment. BS initiated the $\mathrm{CH}$ selection, cluster deployment points are randomly generated as per the average number of clusters.

2. If maximum probability of cluster member for energy, average distance between the cluster members and the number of nodes covered in the cluster region

3. The connectivity cover set formed through the genetic optimization using the location of $\mathrm{CH}$.

4. Cover points forwarding probability computed based on the normalized distributed value of the energy with respect to the distance with the exponential distributed value of the propagation.

5. Do genetic optimization for Connectivity cover set.

6. Cover points forwarding probability computed based on the normalized distributed value.

7. Final cover set is formed to get a maximum coverage. 
2.1.1 Pseudo-code of the Suggested Approach:

BEGIN 1: Cluster deployment started, detect the probability (p),

1.:number of nodes (n);

2: $\operatorname{Einit}(\mathrm{s})=\mathrm{E} 0, \mathrm{~s}=1,2, \ldots, \mathrm{n}$;

Primary Segment

1:Cluster deployment points are randomly generated.

2:Start Counter

3: $\mathrm{CCH}\{\mathrm{s}\}=\mathrm{TRUE}$; //node $\mathrm{s}$ be a candidate $\mathrm{CH}$ with maximum probability function.

4: Direct to Base Station (IDx,) (Xx,Yx) // The first predefine number of node reaches at max probability worth will directs their entry to the sink for cluster head selection.

6: Show To $\mathrm{CH} \leftarrow \mathrm{BS}$ propel message to nominated cluster head.

7: if $(\mathrm{CH}\{\mathrm{s}\}=$ TRUE $)$ then

8: BC (ANC) $\leftarrow$ communicate an announcement message;

9: Cluster(c); // cluster c formation

10: end if

Secondary Segment

1: If Fitness Function $\mathrm{F}(\mathrm{t})>$ true then

2: Apply crossover and mutation

3: Weighted probability estimation

4: Iterative process of the selection, crossover, and mutation

up to the maximum iterations.

5: end if // final chromosome is selected

6: end if // corresponding chromosome is decoded into final cover set.

7:\} // final cover detected

For experimental results shown in table 1, PEAIC and CCOSS is compared for the parameters related to energy efficiency and coverage. Such parameters are, packet delivery ratio, delay in ms, packet dropping, avg. energy consumption, Jitter, Good put, lifetime of network, normalized overhead according to these parameters improvement percentage is calculated.

Table 1 Protocols and results achieved from protocols

\begin{tabular}{|c|c|c|c|}
\hline S.No & Parameter Used & PEAIC & CCOSS \\
\hline 1 & Packet delivery ratio & $92 \%$ & $90 \%$ \\
\hline 2 & Delay(ms) & 37 & 40 \\
\hline 3 & Packet Dropping & 140 & 190 \\
\hline 4 & Avg energy consumption $\left(\mathrm{J}^{*} 10^{-3}\right)$ & 92 & 120 \\
\hline 5 & Jitter J (s) $* 10^{-3}$ & 108.5 & 111 \\
\hline 6 & Good put (b/s) $* 10^{3}$ & 15,000 & 12,500 \\
\hline 7 & Lifetime(s) & 420 & 340 \\
\hline 8 & Normalized overhead & 6.1 & 5.7 \\
\hline 9 & Total Consumed energy $(\mathrm{J})$ & 9,300 & 12,000 \\
\hline
\end{tabular}




\section{Comparision of PEAIC vs CCOSS}

\section{Improvement \%}

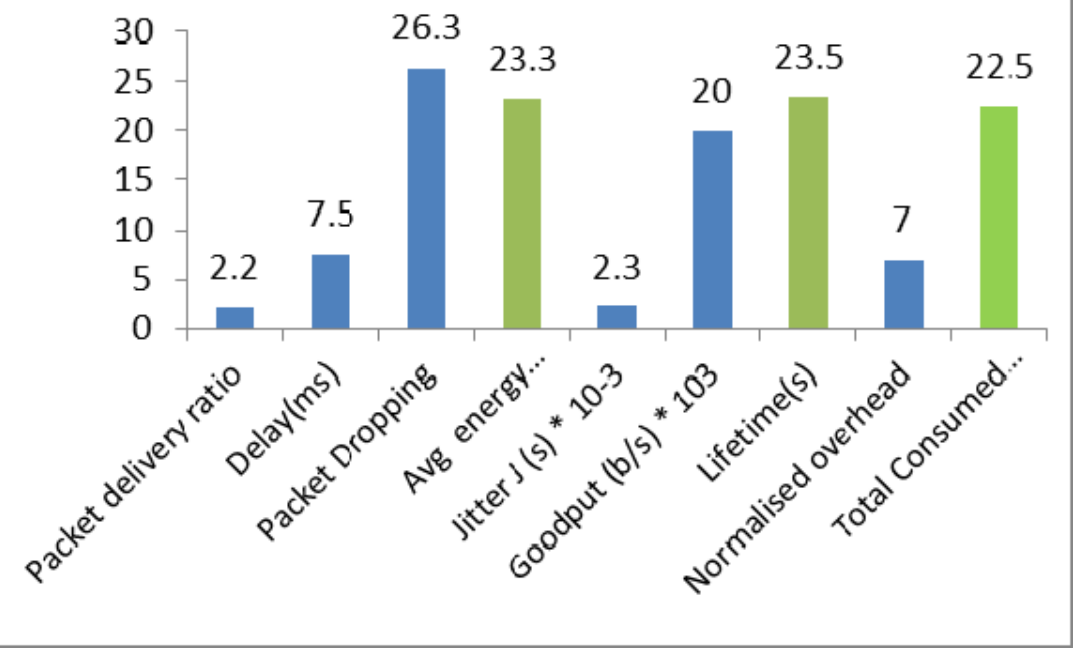

\section{Conclusion}

From the above experiments for comparing PEAIC and CCOSS, we got enhanced performance with respect to packet delivery ratio, Good put, normalized overhead, total consumed energy, Jitter, delay in ms, life time in seconds for PEAIC that is our proposed protocol .The improvement percentage is shown in the graph which proves that for getting more lifetime in wireless sensor networks with maximum coverage is possible by using the proposed Preservation Efficiency and interactive Connectivity (PEAIC) protocol.

\section{References}

[1] Energy Efficient Hierarchical Clustering Approaches in Wireless Sensor Networks: A Survey, BilalJan,1 HaleemFarman,2 HumaJaved,2 BartolomeoMontrucchio,3 MuradKhan,1 andShaukatAli2

[2] A review on energy efficient protocols in wireless sensor networks, Sarika Yadav, Rama Shankar Yadav. The Journal of Mobile Communication, Computation and Information,2015.

[3] A Survey on energy efficient coverage protocols in wireless sensor networks,Avinash More, Vijay Rasininghani,2016, Journal of King Saud University - Computer and Information Sciences.

[4] A new approach for area coverage problem in wireless sensor networks with hybrid particle swarm optimization and differential evolution algorithms, Isa Maleki1, Seyyed Reza Khaze2, Marjan Mahmoodi Tabrizi3, Ali Bagherinia4International Journal of Mobile Network Communications \& Telematics (IJMNCT) ,Vol. 3, No.6, December 2013.

[5] Connected Coverage Optimisation for Sensor Scheduling in Wireless Sensor Networks, Attapol Adulyasas, Member, IEEE, Zhili Sun, Member, IEEE and Ning Wang, Member, IEEE, IEEE Sensors Journal

[6] Probability-Based Coverage Algorithm for 3D Wireless Sensor Networks, Feng Chen, Peng Jiang, and Anke Xue

[7] Deployment of nodes for Maximum Coverage in Heterogeneous Wireless Sensor Network Using Genetic Algorithm, International Journal of Advance Research in Computer Science and Management Studies(IJARCSMS)

[8] Joint Sensor Selection and Multihop Routing for Distributed Estimation in Ad-hoc Wireless Sensor Networks, Santosh Shah, and Baltasar Beferull-Lozano,ieee transactions on signal processing, vol. 61, no. 24, december 15, 2013

[9] An energy-efficient distributed clustering algorithm for heterogeneous WSNs, Javaid et al. EURASIP Journal on Wireless Communications and Networking (2015) 2015:151, A Springer open journal.

[10] Leu, T. H. Chiang, M. Yu, and K. Su, An Energy Efficient Clustering Scheme for Prolonging the Lifetime of WSN With Isolated Nodes, IEEE Communication Letters, Vol. 19, No. 2, Feb.2015.

[11] GWO-LPWSN: Grey Wolf Optimization Algorithm for Node Localization Problem in Wireless Sensor Networks, Rajakumar,R.; Amudhavel, J.; Dhavachelvan, P.; Vengattaraman,T. Journal of computer networks and communications

[12] Lightning search algorithm for solving coverage problem in wireless sensor network, asvany, t.; amudhavel, j.sujatha, p., Advances and applications in mathematical sciences, 2017.

[13] A state of art approaches on energy efficient routing protocols in mobile wireless sensor networks, G.Kadiravan, Pothula Sujatha, J. Amudhave,iioab.

[14] Achieving EnergyEfficiency and Increasing the Network Life Time in MANET through Fault Tolerant Multi-Path Routing, February 25, 2017, NandithaBoddu,Ramesh Vatambeti, VeeramalluBobba.

[15] Adaptive data transmission in WSN using enhanced path assured transmission protocol, Avinash Devare, G. K. Mohan, Hruturaj B. Nikam.

[16] Multi-level Structured Tree based Routing for Energy Efficiency in WSN, Dr. Thirupathi Regula, Dr. Mohammed Ali Hussain,International Journal of Engineering \& Technology.

[17] Optimization of Energy Aware Path Routing Protocol in Wireless Sensor Networks, M. Naga Vamsi Krishna, N. Sai Harsha, K. V. D. Kiran, Gandharba Swain. 
[18] Comparative study of leach and pegasis energy efficient Protocols, Pakam Sundaraiah,K Sri Kapardi, CH Jaya Lakshmi, K Srinivas, M V Uday Kumar, Journal of Advanced Research in Dynamical and Control Systems

[19] Architectural analysis for lifetime maximization and energy efficiency in hybridized WSN model, Kale Navnath Dattatraya, K. Raghava Rao, D.Satish Kumar, International Journal of Engineering \& Technology.

[20] A Comparison Review on Comb-Needle Model for Random Wireless Sensor Networks, M. Shanmukhi, J.AmudahavelA. VasanthiG. Naga Sathish, 2019.

[21] Investigation on maximizing packet delivery rate in WSN using cluster approach. Wireless Personal Communications,T.Karthikeyan,V.Brinda,P.Manilagai. 